\title{
Research on Communication Technology of Smart Distribution and Utilization Network
}

\author{
Qihao Zhang ${ }^{a}$, Yanping $\mathrm{Li}^{\mathrm{b}}$, Liting Wang, Caifeng Li \\ State Grid Henan Electric Power Company Luoyang Power Supply Company, Luoyang 471900, \\ China \\ azhangqihao0@163.com, b1015903240@qq.com
}

Keywords: Communication technology, distribution and utilization network.

\begin{abstract}
At present, China's smart grid construction has been in full swing, with the continuous development of smart grid technology, its business application systems with electric side is also gradually improved, to respect the distribution communications network bandwidth and higher reliability requirements. With electric communication network is an important part of power communication network platform, is the power backbone communications network extends downwardly This paper analyzes the intelligence with comprehensive analysis of the characteristics and business development needs of the communication network of electricity from a technical, economic aspects and compared and combined with practical application scenarios and channel conditions proposed system architecture and networking technology solutions intelligent distribution, power communication network.
\end{abstract}

\section{Introduction}

Smart Grid is the latest development trends of the world's electric power system reform, which include generation, transmission, transformation, distribution, use and scheduling of the various aspects of the power system as an object, continuous research and development and application of new control technology, information technology and management techniques, intelligent exchange of information. Communication and information systems as a support smart grid construction and an important means of public platform, runs through the six application link.

Communications and Information under the trend of intelligent power grid construction, will promote the realization of network planning, design, construction, production, gathering comprehensive operational, service information, transmission fluid and efficient handling, lifting equipment, and business process automation level is formed include the company's entire business information systems, enhance the level of modern business management, to optimize the whole network resource allocation, efficient use and comprehensive risk control structures transparent sharing of information, process specifications integrated, powerful business collaboration and interoperability platform to enhance the human and application systems between, among the six links between business, the company and the level of interaction between the various stakeholders take advantage of a strong smart grid pluralism, the potential value of the mass of information, and tap their knowledge behind it, enhance intelligence analysis grid and scientific decision-making level of the use of smart grid to a variety of power supply, electrical equipment connected to one, to provide communications support networking, to expand networking applications.

\section{Analysis of Business Requirements}

Distribution automation systems supporting the construction of distribution and communication network are mainly implemented in the city, there is no unified plan of distribution communications network. Communication from the implementation of distribution automation system development overall, and the developed eastern coastal cities is better, the coverage is relatively high in the western region only a few pilot projects, with lower coverage. And a general statement, distribution automation and supporting communications systems are still in the small-scale application stage, it 
has not yet set up to build a large-scale expansion. Currently, the distribution network for multiple use many communications ,for example, optical modem communications, industrial Ethernet, Ethernet passive optical network, medium voltage power line carrier, wireless public network, and other communications.

\section{Analysis of Distribution Traffic.}

Distribution Automation system is mainly the acquisition and control switching station, ring counters, box-type substation, column switch, pole-mounted transformer and other equipment information. Preliminary consideration of the current distribution network an important node in the opening and closing operation of the station and monitoring requirements, consider each important node configured video surveillance business, to run the business channel, and voice services. Each distribution line an average opening and closing consideration quality video services image formats to meet the requirements to consider traffic is. To run the business channel primarily serve local operation ticket, receive and transfer needs work tickets, traffic. Distribution Automation system involves control switchgear in safe areas, in accordance with the relevant safety standards, in order to ensure the safety and reliability of control should be used exclusively way communications network communications network should have high reliability and redundancy.

In the present communication in accordance with the conditions established distribution network technical specifications, in the private network communication condition to the master switch quantitative bit less than 10 seconds, change the telemetry transmission time is less than 20 seconds, the sequence of events recording resolution of less than one second, electricity Energy scan cycle 5 minutes. In the public network communication condition to the master switch quantitative bit less than three minutes, telemetry (the dead zone) change the transmission time of less than three minutes, the system required to achieve the same technical indicators point should gather information uploaded by the sub-station in.

\section{Analysis of Electric Traffic.}

Operational characteristics of electricity mainly in the business of communication nodes and more diverse business categories, the flow of centralized information collection requirements focus on the collection at a predetermined point in time all of the user's data metering equipment to monitor and manage real-time information occurs immediately active electricity billing data upload, remote control instruction information randomness, real-time requirements, information flow is small interactive electricity service class information for bidirectional communication have higher requirements, end users will have the operational characteristics of voice, data and video communications. Thus, in the power communication network with variable data and meter node access node is traffic bottlenecks.

Information gathering information collection system is huge, wide coverage, and safety-related electrical power users, its collection, customer information, the power of information and tariff information transmission, storage, handling and use only the core data and other power industry information, and involve all levels of the country's political, military and social life, with high confidentiality, illegal use and leakage of information may bring irreparable damage. So the network information security to be achieved on the basis of the original acquisition system on a higher level in order to achieve security requirements for the State Grid Corporation overall information system providing accurate, reliable, efficient, real-time data base marketing business applications.

\section{Equipped with Select Smart Grid Communications Technologies}

Distribution, electric power communication network is a backbone communication network extends downward, and is the backbone of the network access layer down to cover the site with all levels of the grid, and user energy meter indoor communication terminals, and distribution of electric vehicle charging stations type energy sites and other related equipment. Integrated distribution, electricity demand forecasting communication, analysis and calculation of real-time traffic and safety with electricity distribution network to the end of the communication network and user meter 
boundary demarcation point, into the distribution communication network, electricity connection network and customer premises network three levels.

\section{Network Architecture.}

Currently with the main business communications network carrying the electricity distribution automation business and business information collection system, from a business perspective, distribution automation system communication network into a communications network backbone layer and access layer communications network, wherein the backbone layer communication Network implement distribution master station to communicate with the electronic correspondence between power communication optical fiber backbone network and access layer with an electronic communications network mainly realizes the power distribution station to the communication between the terminals corresponding to the power distribution communications network, with electric communication network information collection system is divided into a telecommunications network and wooden ground communications network, wherein the telecommunications network for communication power between the main station and the concentrator, which corresponds to the electricity distribution network and optical fiber communication backbone communications network, local communication networks and communications concentrator and meter collector between the corresponding user access network.

\section{Technical Options.}

Distribution network communications network for security, reliability and high bandwidth requirements, should adopt special network power mode optical fiber-based, wireless broadband private network and medium-voltage power line carrier communication technology as a supplement, cannot cover in some areas Power Communication Network, communications operators may be public network communication mode as a supplement, but the public network communication mode should be limited to not require remote control terminal communication applications. As traditional, high transmission standard investment, long construction period, to be occupied by the engine room and power supply, operation and maintenance of large disadvantages, PON system with its unique technical advantages as the preferred distribution automation site information access methods. PON system using high light ratio, it is possible in a relatively short time to target regional distribution network to achieve rapid coverage. PON system network to be a better solution supporting optical cable construction, and reasonable arrangements for splitting the network configuration. To build or renovate distribution lines, fiber optic cable can be OPLC of old lines, should be set up or general ADSS cable. According to the distribution network of information points with the distribution network lines linear series of characteristics, ODN network should adopt unequal splitters to ensure network flexibility and scalability.

The user access network construction, the need to achieve a two-way interactive marketing services, smart home, users value-added services, to higher channel bandwidth, real-time, safety and reliability requirements, priority should be the private network communication technology using optical fiber or broadband over power line communications technology, only need to implement the information collection system business users can use the power line broadband communications, narrowband power line communications or micro-power wireless technology. New Intelligent Community of communication using Ethernet passive optical network technology, customer premises ONU terminal configuration, user interactive terminals, intelligent set-top boxes, IP phones, computers and other devices via Ethernet interface and ONU terminal connectivity for voice, data, cable TV information access.

Customer premises network will be formed between the user and the electrical equipment air conditioners, washing machines, water heaters and other network interaction and instant connectivity for interactive service for electricity, the Internet Gateway distributed energy management, smart home and security, and other value-added services. Customer premises network for power line networking technology broadband communications technology, power line narrow-band communication, and micro-power wireless technology. Power line broadband communications technology because of high bandwidth, moderate cost, no separate wiring, electromagnetic radiation, etc. are suitable for the formation of customer premises network technology of choice for networking 
solution, micro-power wireless technology and narrowband power line communication technology is also optional technologies.

\section{Summary}

With electricity communication network construction is an important part of smart grid construction; it is the full realization of intelligent distribution, the power of information technology, automation and interaction of important support and protection. With electricity and with communications applications, with simultaneous development of intelligent network services and value-added business needs and moderate advance, at the same time, we should focus on the development of power unique communication technologies. The public is limited to its technical and operational characteristics, can be used as electricity private network is temporarily unable to cover the necessary complement to the node.

\section{Reference}

[1]Xin P, Jun L I, Wang Y, et al. Communication Technology and Application Scheme of the Smart Distribution Grid[J]. Electric Power Construction, 2011.

[2]Xin P Z, Jun L I, Wang Y D, et al. Research on Communication Technology in Smart Distribution Grid[J]. Telecommunications for Electric Power System, 2010.

[3]Jian-Ming $\mathrm{H}$ E. The application of wireless communication technology in the intelligent distribution network[J]. Technological Development of Enterprise, 2011.

[4]Liu G, Hou X, Wang N, et al. An Integrated Network Management System for Communication of Smart Power Distribution and Utilization Grid[J]. Power System Technology, 2012, 36(1):12-17.

[5]Dai W, Chen H, Wei X U. Research of Smart Distribution Network Based on EPON Communication Technology[J]. Telecommunications for Electric Power System, 2012. 